\title{
Notizie riassuntive sui risultati delle ricerche nella Grotta di Paina sui Colli Berici (Vicenza, Italia)
}

\author{
Piero Leonardi *
}

La Grotta di Paina —chiamata localmente Covolo di Paina - ha preso il nome da una vecchia famiglia di contadini che se ne serviva per attività inerenti alla pastorizia e abitaba la casetta che ne ostruisce per tre quarti l'ingresso.

Essa (figg. 1, 2), scavata in calcari paleogenici appartenenti ad un' antica scogliera coralligena, è situata nel Vicentino poco sopra il paese di Mossano, a m. $335 \mathrm{s.m}$. al centro di quella che viene chiamata, per la sua morfología e per l'ottimo clima che favorisce nella sua flora elementi mediterranei, la "Riviera Berica".

E'questa rispondente ad una lunga scarpata con numerose paretine rocciose, rivolta a Est - Sud - Est, dovuta a una grande frattura, una delle maggiori entità tettoniche del Vicentino.

La Grotta di cui parliamo - pur essendo una delle maggiori tra quelle esistenti nei Berici (M. 42 di lunghezza X m. 8 di larghezza massima)era poco nota nell'ambiente speleologico e paletnologico - benché scoperta nel $1939^{1}$ - finché, un trentennio fa, essa venne segnalata allo scrivente dallo scopritore, il carissimo Amico Aldo Allegranzi, che stava

* Membro della "Academia de Ciencias" de España.

'La scoperta, da parte di A. Allegranzi, venne resa nota da G. Trevisiol nel 1940 (vedi bibliografia). 
per diventare uno dei suoi più preziosi collaboratori in quel campo di ricerche.

Da allora —da quando cioè ebbero inizio, nel 1957, gli scavi sistematici nel suo deposito di riempimento, tuttora in corso- la Grotta di Paina entrò a far parte di quello sciame di cavitá dei Colli Berici che gode attualmente di un posto di rilievo nello studio della Preistoria del Veneto.

Certo non si può dire che la nostra grotta meriti - per la potenza del deposito o per la richezza di reperti- una rinomanza paragonabile, per esempio, a quella della Grotta del Broion neu medesimi Colli Berici ${ }^{2}$, oppure - per la scoperta di notevoli opere d'arte mobiliare paleoliticaparagonabile a quella del Riparo Tagliente nei Monti Lessini ${ }^{3}$ - ma non si può negare che anch'essa meriti una certa considerazione per il valore eccezionale di certi manufatti epigravettiani, e per il fatto che essa ha il privilegio di possedere una delle piú complete serie stratigrafiche del Paleolitico Superiore del Veneto.

Come si diceva, la grotta è scavata in rocce calcaree che hanno dato luogo a fenomeni carsici di grande rilievo, che nel caso specifico hanno avuto origine dalla esistenza, lungo l'odierno asse principale della caverna, di una potente diaclasi, valendosi della quale una corrente d'acqua di superficie si aprì il varco nel profondo della massa rocciosa elaborando l'attuale enorme cavità e depositando in essa una parte rilevante del deposito che forma oggetto delle nostre ricerche.

La grotta si compone di tre vani largamente comunicanti: una grande cavitá, chiamata "Sala Grande" (fig. 1), con scarso rivestimento di concrezioni (che, pur assumendo que e là conformazioni esteticamente abbastanza piacevoli, non raggiungono mai le caratteristiche di vere stalattiti), il cui fondo è stato, nella parte anteriore, non soltanto svuotato dal deposito di riempimento, me addirittura approfondito con l'escavazione della roccia sottostante (fig. 2), probabilmente allo scopo di ricavare i materiali per costruire la casetta e forse anche il muraglione di sostegno del piazzale antistante. A circa due terzi della lunghezza della grotta si ha una strozzatura per il restringimiento delle pareti e l'abbassamento della volta e si entra in una seconda cavità minore detta "Sala Terminale" (fig. 3), alla cui estremità interna sale un "camino" verticale alto $7 \mathrm{~m}$. (fig. 2), ora chiuso superiormente, ma probabilmente un tempo apren-

2 P. LeONARd, Riv. Sc Preist., Vol. VI, e varie altre pubblicazioni. 1951.

${ }^{3}$ P. Leonardi, Riv. Sc. Preist. Vol. XXVIII, e varie altre pubblicazioni. 1972. 


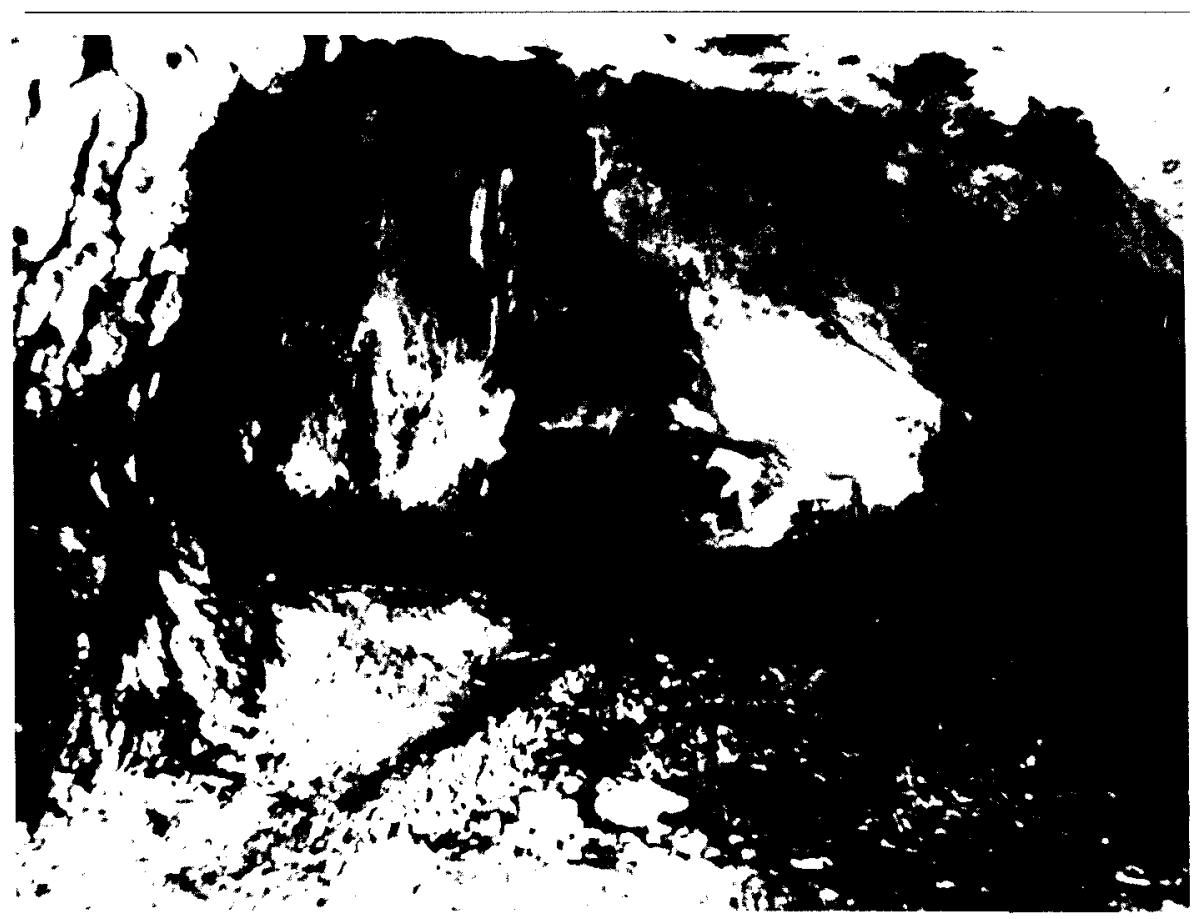

Fig. 1. Veduta generale della Grotta di Paina. In fondo alla Sala Grande, lingresso della Sala Teminale. Un po' più a destra, illuminata per gli scavi in corso, si apre la Grottina Azzurra. In primo piano, al di qua del gradino sassosso, la zona più esterna della grota, in cui il suolo è stato approfondito anni fa per ricavare materiali per la costruzione della casetta antistante. Cfr. fig. 2. (Fotografía di DANIELE ROSSI).

tesi sul fondo di una qualche "dolina", ed dal quale scendeva un rivo cui è in parte dovuto in deposito alluvionale della grotta. Infine, presso il passaggio fra le due cavitá suddette, sulla destra entrando, c'è una nicchia di modeste dimensioni, chiamata "Grottina Azzurra» (fig. 2) perchè le sue pareti e la volta presentano una tinta appunto azzurra per essere rivestite da uno straterello di cianoficeae, ora ridotto, perchè il restringimento dell' ingresso ha ridotto la luce che entra nella grotta.

In una prima fase di scavo (1957-58) si sono praticate delle trincee nella sala terminale, nella Grottina Azzurra a nella Sala Grande in prossimità dell'ingresso di quella.

In una seconda fase, iniziata nel 1981 a tuttora in corso, si sono estesi e approfonditi gli scavi, esaminando e rapporti intercorrenti tra le serie stratigrafiche dei vari ambienti. 


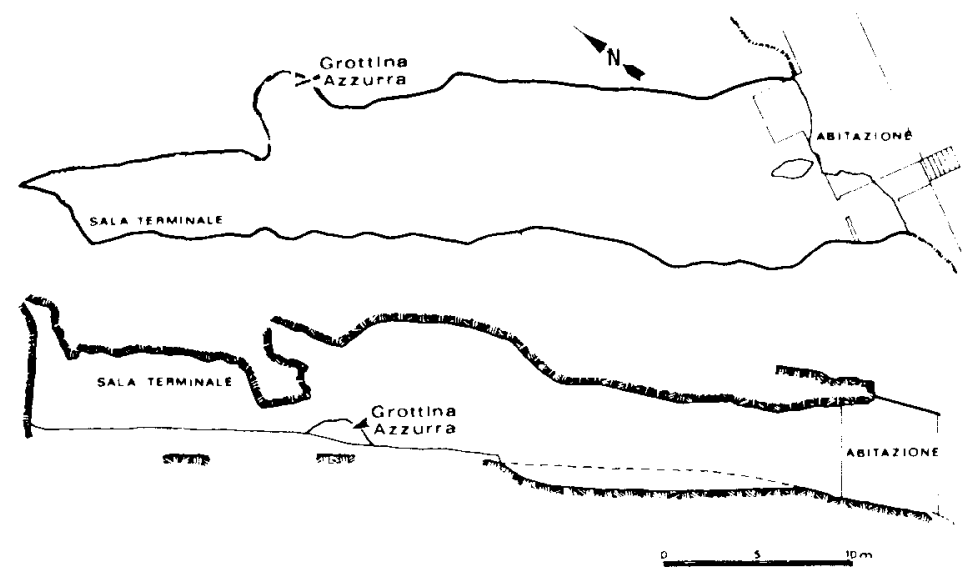

Fig. 2 Planimetria e sezione longitudinale della Grotta di Paina. La linea tratteggiata a destra della sezione indica il livello originario della superficie rocciosa di base prima degli scavi per ricavare materiali per la costruzione della casetta. (Da A. Broglio, 1988).

Il deposito di riempimento della serie della Grottina Azzurra è costituito prevalentemente da materiali alluvionali argillosi e sabbiosi, più o meno ricchi di pietrame, depositati dal rivoletto cui si è accennato, e da numerosi blocchi di crollo anche di grandi dimensioni, specialmente al passaggio tra le due sale, con intercalazioni di sabbie eoliche loess a straterelli di guano.

Ad un certo livello entro il deposito della Sala Terminale c'è un letto di breccia tenacemente cementata e molto elaborata in superficie dall'acqua, che nella prima fase delle ricerche era stato interpretato come la roccia di fondo della grotta, mentre la sua vera natura è stata chiarita nella fase successiva tuttora in corso, nella quale è stata scavata in varii punti anche la parte del deposito sottostante.

Le indagini palinologiche, dovute a Laura Cattani ${ }^{4}$ hanno permesso di distinguere nella serie della Grottina Azzurra due cicli climatici, uno corrispondente alla parte inferiore del deposito (strati 12-10), di tipo continentale con sopravvivenza di un modesto consorzio arboreo a latifoglie, mentre la parte superiore (strati 9-5) risulta rispondente ad un ciclo arido freddo caratterizzante un ambiente steppico specialmente in corrispondeza dello strato loessico 6 , che denota un ambiente periglaciale.

${ }^{4}$ L. CatTANI, in AAVV (1987-88) 1989. 

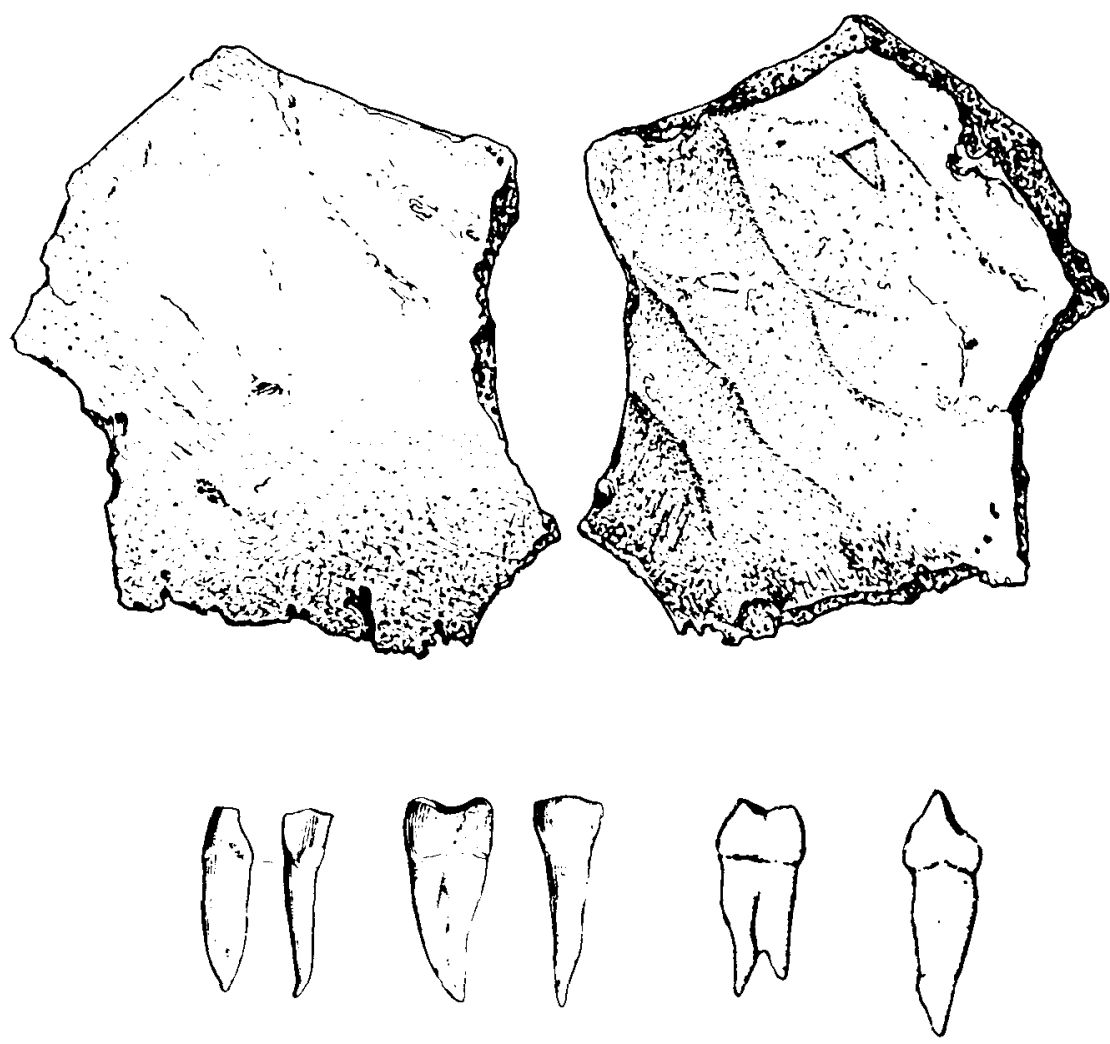

Fig. 3 Frammento di parietale e denti umani rinvenuti nella parte superiore -rimaneggiata - della serie della Grottina Azzurra. I due denti a sinistra (incisivo $e$ premolare) provengono dagli scavi 1957-58; i due a destra (premolare e canino) dagli scavi recenti. Grandezza naturale. Disegni di B. SantochI e G. Almeri. gogna. (Da P. Leonard et allii, 1962 e da A. Broglio, 1988).

I reperti faunistici sono abbondanti sia per quanto riguarda i macro - che i micromammiferi. Tra i primi predomina decisamente l'Orso speleo il quale evidentemente aveva nella Grotta di Paina un suo ricovero abituale, come è dimostrato oltrechè dall'abbondanza dei suoi resti, che comprendono tutte le parti del corpo, anche dal fatto che essi sono riferibili a individui di tutte le età, a partire dagli infanti, i cui denti minuscoli e assai caratteristici sono particolarmente frequenti.

A probabili prede di caccia da parte dell'uomo sono invece riferibili gli avanzi di altri macromammiferi come il Leone, la lena, il Gatto selva- 
tico, un bovide, un equide e il Cinghiale, rappresentati per lo più da dentie e ossa di metapodi isolati, e i frequenti erbivori, tra i quali predominano il Cervo e l'Alce, mentreè è raro lo Stambecco.

Quanto ai micromammiferi, roditori e chirotteri, essi sono abbastanza ben rappresentati e provengono con ogni probabilità dai "boli» o rigetti dei rapaci, ancora fino a pochi anni fa molto abbondanti sui dirupi della costiera circostante.

Concordano sostanzialmente con quelli dello studio palinologico i risultati dello studio dei reperti faunistici, dovuto a Giorgio Bartolomei ${ }^{5}$. Gli strati basali (12-10) rispondono a una prateria arborata continentale moderatamente umida, lo strato 9 testimonia un brusco cambiamento con spostamento verso un ambiente montano e umido e più fresco d'estate, e negli strati 7-5 si assiste al passaggio a condizioni steppiche, che favoriscono la deposizione del Loess e sono riferibili per il contesto paletnologico al $1 \mathrm{I}^{\circ}$ Pleniglaciale würmiano. Quanto allo strato 4 - se il suo contenuto faunistico è autentico, e cioè non inquinato per cause varie da elementi provenienti da strati sovrastanti- sembra rispondere ad una oscillazione umida del Tardiglaciale.

Gli scavi finora conditti nella Grotta di Paina non hanno fornito granchè in fatto di resti scheletrici umani.

Quel poco che si è trovato proviene dai livelli superiori del deposito della Grottina Azzurra.

Si tratta innanzitutto di un frammento cranico appartenente al parietale sinistro (fig. 3), rinvenuto durante assaggi preliminari compiuti da $A$. Broglio e E. Menaldo nel 1956. Uno dei suoi lati corrisponde al margine sagittale e mostra in parte conservate le denticolazioni della sutura omonima. La superficie interna mostra chiaramente le terminazioni superiori dei solchi corrispondenti a varie rami dell'arteria meningea media.

Sempre nella parte superficiale del deposito della Grottina Azzurra sono stati trovati quattro denti: due negli scavi iniziali (1957-1958) e due nel corso delle ricerche più recenti. Dei primi due uno è un premolare superiore, probabilmente un primo premolare, e il secondo un incisivo laterale destro. Sono ambedue assai ben conservati (fig. 3 a sinistra). Gli

${ }^{5}$ G. BartolomeI in AAVV (1987-88) 1989. 

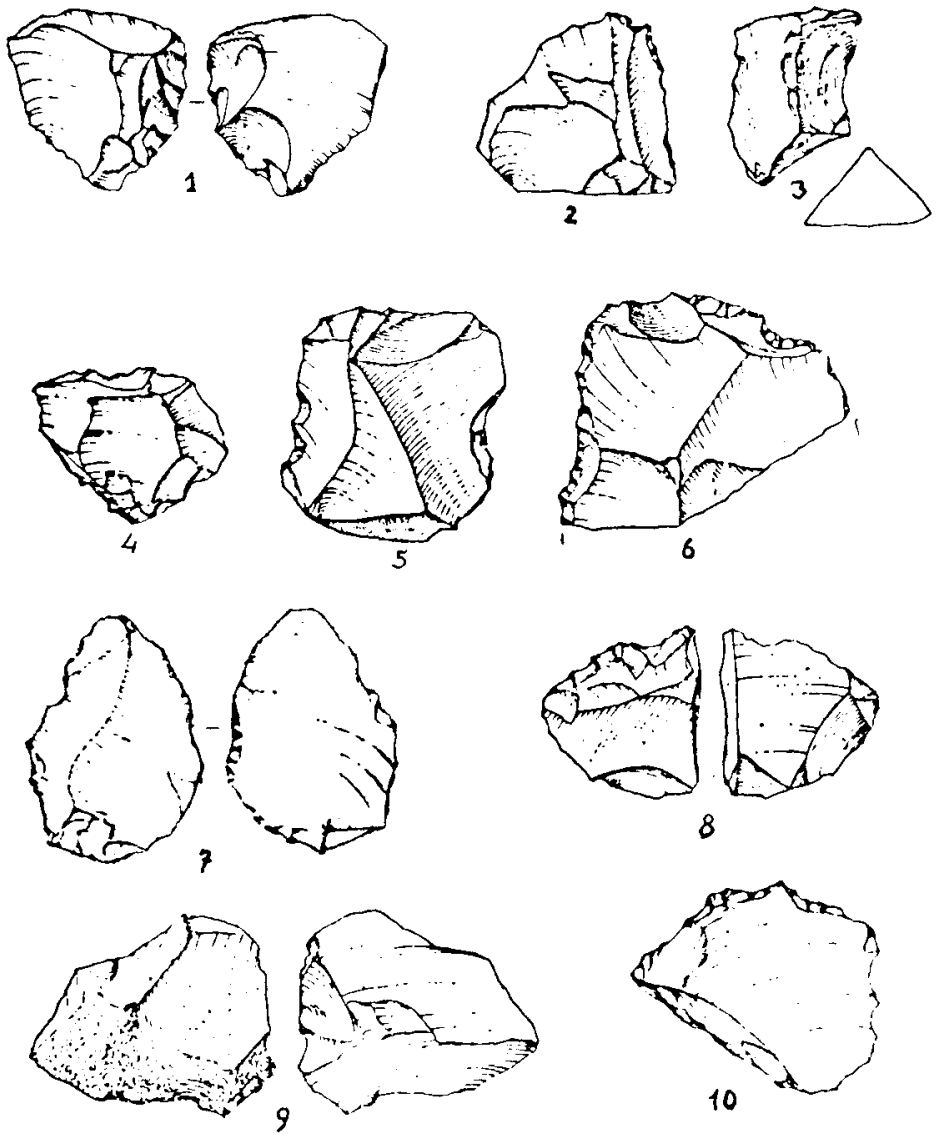

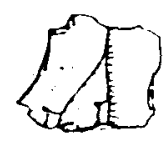

11

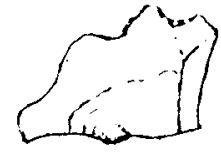

12
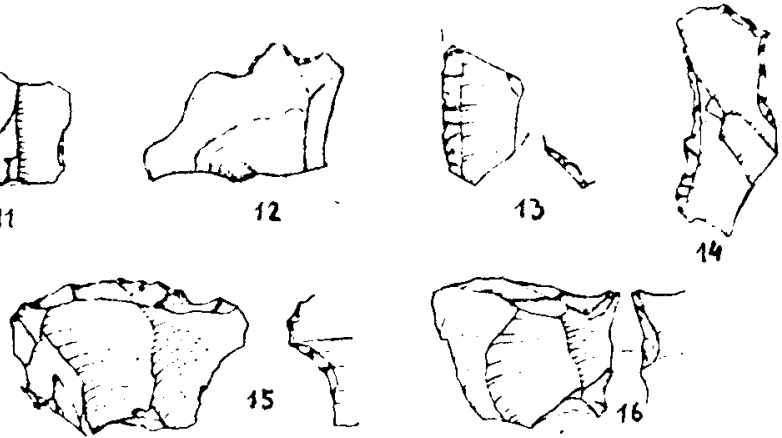

Fig. 4. Industrie pre-aurignaciane (Mustieriano ?) degli strati 10 (N.' 1, 4-6); 11 (N.' 2, 3); 12 (N.' 7-16). Disegni di G. Almerigogna. (Da A. Broglio e N. LanzinGER in: AAVV [1987-88] 1989). 
altri due, rinvenuti successivamente, sono un canino probabilmente inferiore e un primo molare superiore a sviluppo completo (fig. 3 a destra).

E'difficile dire se i quattro denti e il frammento cranico appartengono ad un medesimo individuo, il che d'altra parte non si può escludere. Ancor più difficile è stabilirne l'età, data la natura del deposito in cui sono stati rinvenuti, che ha subito rimescolamenti vari e contiene elementi faunistici e culturali eterogenei.

I resti qui descritti non differiscono in nulla dalle parti corrispondenti di Homo sapiens attuale, ma lo stato di conservazione, del tutto analogo a quello dei reperti faunistici pleistocenici, farebbe propendere a ritenerli, sia pur con molte riserve, paleolitici.

Per quanto riguarda la successione culturale, la serie stratigrafica della Paina (pur nel suo limitato spessore) è abbastanza vasta. Negli strati più profondi (12-10) al limite tra la Grottina Azzurra e la Sala Grande l'ultima campagna di scavo (ottobre 1988) ha rinvenuto qualche manufatto piuttosto atipico, ma che farebbe pensare alla possibile presenza del Mustieriano (fig. 4).

Lo strato 9 ha fornito due lamelle Dufour e un frammento mediale di zagaglia d'osso piatta (fig. 5) riferibili all'Aurignaciano ${ }^{6}$.

Nello strato 7 si rinvennero alcuni frammenti di punte a dorso, tra cui la base di una punta dei Vachons (fig. 6) riferibili al Gravettiano ${ }^{7}$.

I reperti paleolitici più interessanti della Grotta di Paina sono rappresentati da un gruppo abbastanza nutrito di punte a cran (fig. 7) provenienti dallo strato 6 specialmente della Grottina Azzurra, e in quantità minore dal deposito della Sala Terminale. Esse sono riferibili senza dubbio all'Epigravettiano italico antico. Come riferisce A. Broglio "Esse sono ricavate da lame di dimensioni relativamente grandi; negli esemplari integri la lunghezza varia da 65 a $90 \mathrm{~mm}$, la larghezza da 10,5 a $14,5 \mathrm{~mm}$, l'indice di allungamento da 4,2 a 6,5. La morfologia è varia. La purita è ottenuta mediante due ritocchi erti marginali convergenti, oppure mediante un dorso profondo continuo o ancora (in un solo esemplare ricavato da una lama spessa) mediante ritocco semplice. II cran è sempre ricavato

\footnotetext{
${ }^{6}$ A. Broglio, 1988, pág. 62.

Ibidem.
} 

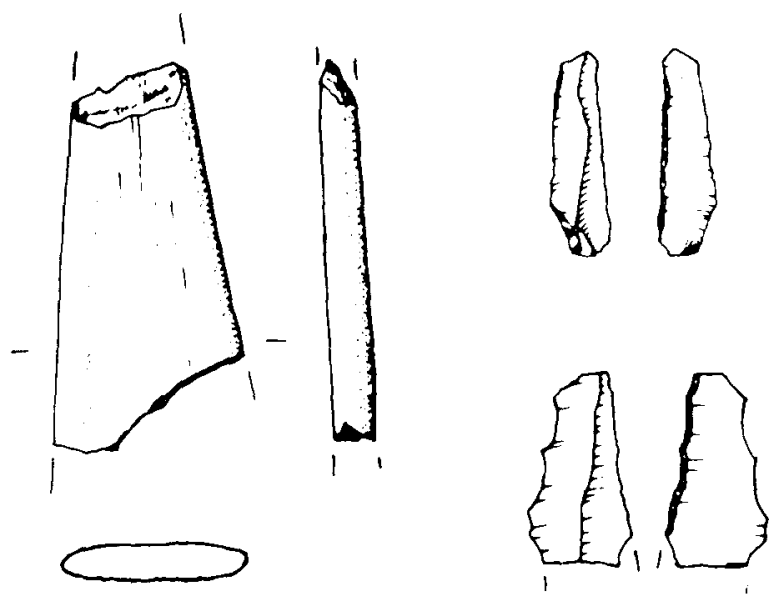

Fig. 5. Industria aurignaciana dello strato 9 della Grottina Azzurra: frammento di zagaglia d'osso e lamelle Dufour. Grandezza naturale. Disegni di G. ALmERIgogna. (Da A. Brogloo, 1988, pág. 61.)
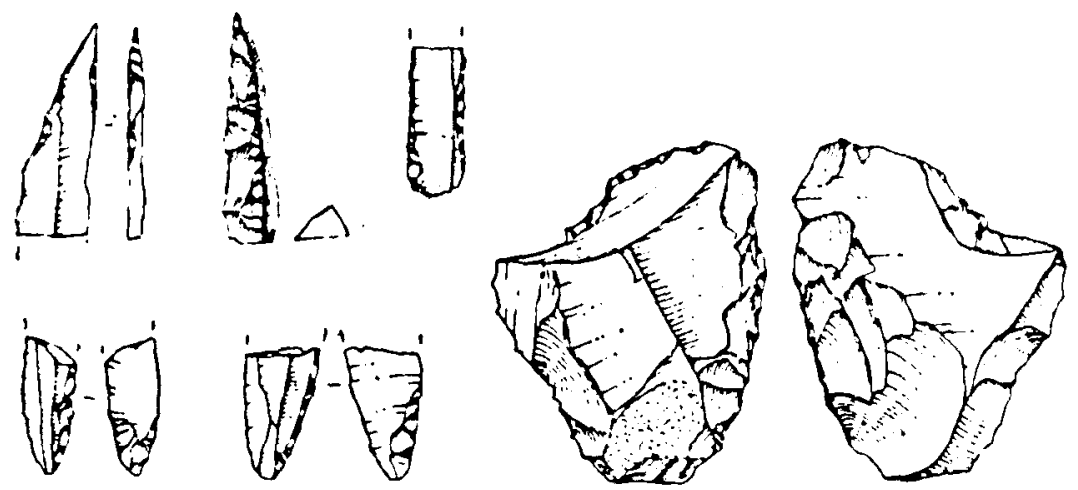

Fig. 6. Manufatti gravettiani dello strato 7 della Grottina Azzurra. Grandezza naturale. Disegni di G. Almerigogna. (Da A. Broglio in: AAVV, [1987-89] 1989). 

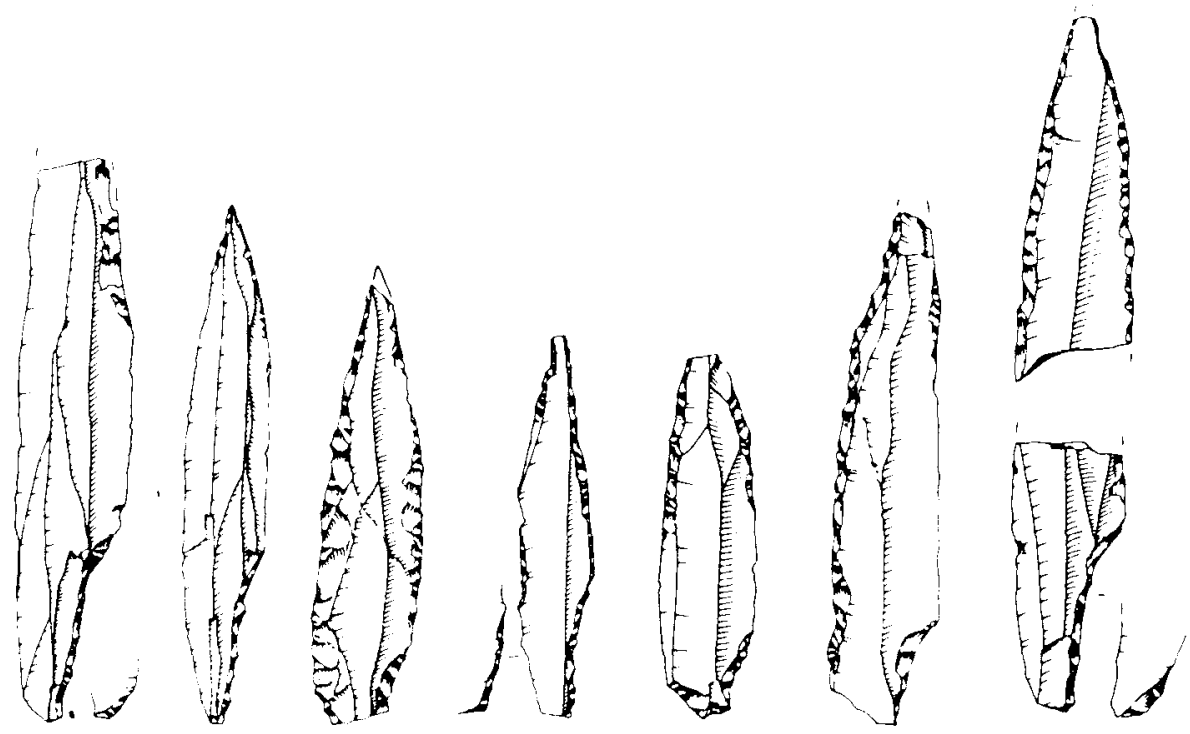

Fig. 7. Serie di punte a cran epigravettiane dello strato 6 della Grottina Azzurra, salvo il penultimo esemplare a destra, che proviene dall'area antistante al suo imbocco, e l'ultimo, pure a destra, che proviene dallo strato $C$ della Sala Terminale (Scavi 1957-58). Grandezza naturale. Disegni di G. Almerigogna. (Da A. Broglio, 1988, pág. 60.)

mediante ritocco erto profondo ed è di lunghezza media (oltre $20 \mathrm{~mm}$ ) tranne che in un pezzo, nel quale è corto" ${ }^{8}$.

Questo gruppo di punte a cran è senza dubbio il più notevole, sia qualitativamente che quantitativamente, conosciuto finora nei giacimenti del Veneto.

$\mathrm{Va}$ ' rilevato, in senso ancor piú generale, che addirittura, secondo A. Broglio (1984, 1987-88) l'Epigravettiano italico antico a crans come complesso sarebbe noto - nell'Italia padana - soltanto nel deposito della Grotta di Paina. Un esemplare sporadico rinvenuto in superficie è stato segnalato dallo scrivente sul Castellon del Brosimo, non molto lontano dalla Paina, assieme a manufatti più recenti ( $P$. Leonardi, 1951, tav. IV. ${ }^{\circ}$, fig. 15; A. Broglio, 1987-88, 1989; fig. 20).

${ }^{8}$ Ibidem. 
Quanto all'Epigravettiano italico recente, esso è pure rappresentato nella Grotra di Paina ${ }^{9}$, ma molto più scarsamente, nello strato 5 della Grottina Azzurra, da cui provengono solo pochi frammenti di strumenti ad dorso e di una punta d'esso (fig. 8).

II Mesolitico è ancora più scarsamente rappresentato da armature microlitiche di tipo sauvetterriano rinvenute nella parte superiore rimaneggiata della serie della Grottina Azzurra e forse in posto nella Sala Grande ${ }^{10}$. Un tipico microbullino (fig. 9) era stato trovato dallo scrivente nella prima fase degli scavi, sempre nei livelli superiori rimaneggiati ${ }^{11}$. Infine nei livelli superiori della Sala Terminale sono associati manufatti eterogenei (lame e nuclei di selci di età varia e qualche frammento ceramico riferibile al Calcolitico ${ }^{12}$, al quale è da attribuire anche qualche manufatto siliceo o fittile (fig. 10) dello strato 4 (strato A. dei vechi scavi) della Grottina Azzura ${ }^{13}$ e dello strato A della trincea dei vecchi scavi antistante alla medesima. Tra quest' ultimi figura una punta di freccia probabilmente riferibile al Calcolitico (fig. 11).

I reperti suindicati erano in prevalenza associati nella Sala Terminale al tipico focalare con carboni, ossa bruciate e manufatti litici e fittili. Questo focolare è l'unica testimonianza di abitazione nella grotta con una relativa stabilità. Ciò è confermato anche -come osserva giustamente $A$. Broglio (1987-88), 1989, riassunto) — «dalla struttura degli insiemi litici del Gravettiano e dell'Epigravettiano, che suggerisce che la Grotta sia stata utilizzata come campo ocasionale di caccia».

Non si può dire che fino al 1938 fossero state rinvenute nei giacimenti paleolitici dei Colli Berici manifestazioni grafiche, se non si voglia far uso di questa qualifica a proposito di un manufatto osseo subcilindrico, ricavato da una diafisi di osso lungo, con varie fini inicisioni trasversali con ogni probabilità intenzionali (fig. 12a). Rinvenuto nella Grotta di Trene, sempre sui Colli Berici, al limite tra gli strati A' e A" ma con ogni probabilità riferibile al Paleolitico Superiore ${ }^{14}$, e di un frammento di os penis di Orso (speleo?) rinvenuto nella parte superiore rimaneggiata della Grottina Azzurra della Paina, il quale si mostra levigato per l'uso e reca

\footnotetext{
9 A. Broglio, pág. 63. 1988.

10 A. Broglio, pág. 651988.

P. LeONARDI, pág 2, fig. 1; AAVV (1987-88) 1989, FIG. 15/13. 1957

AAVV, (1987-88) 1989, pág. 4.

13 A. Broglio in: AAVV, 1962, pág. 103, fig. 15.

${ }_{14}$ P. LEONARDI, pág. 34, fig. 34. 1959.
} 

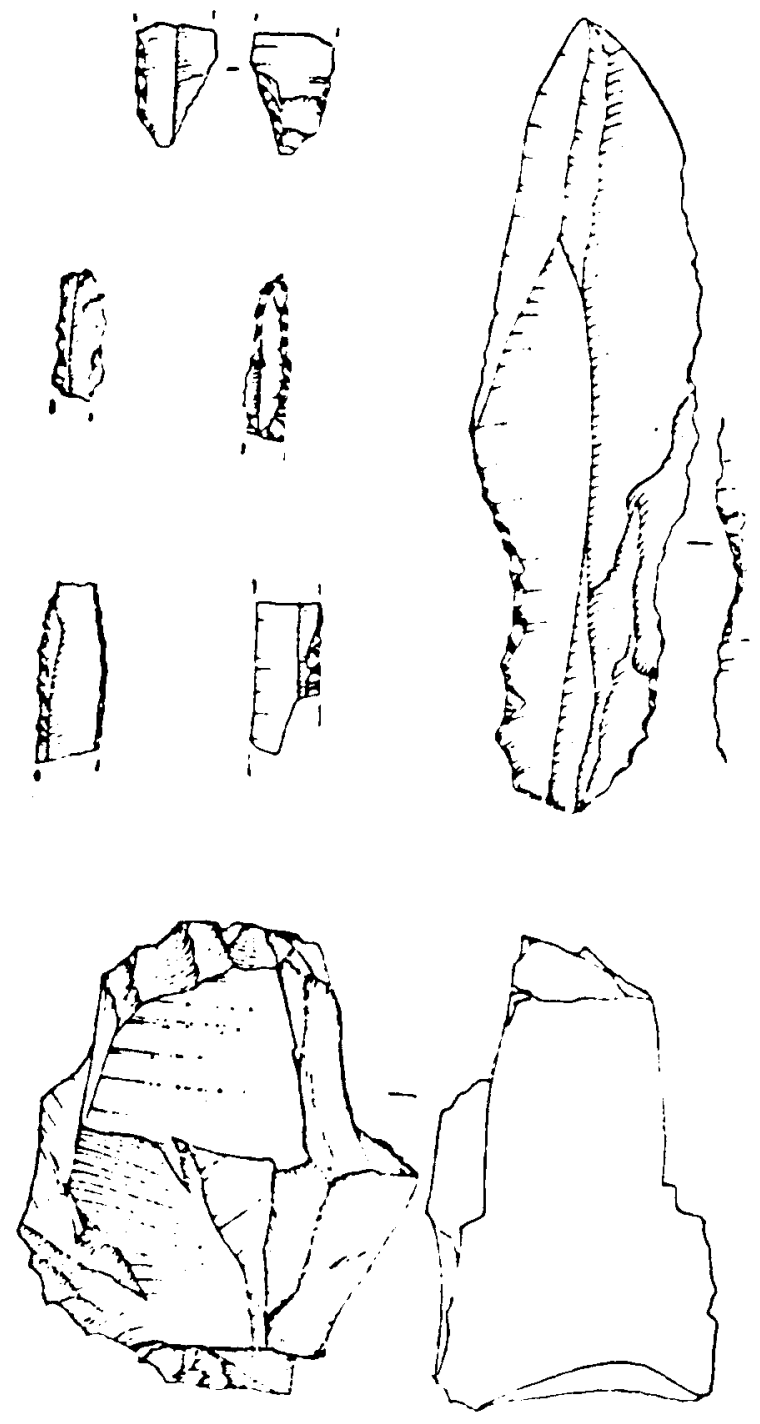

Fig. 8. Manufatti silicei riferibili all'Epigravettiano recente, provenienti dallo strato 5 della Grottina Azzurra. Grandezza naturale. Disegni di G. Almerigogna. (Da A. Broglo in: P. LEONARDI et alii, 1962). 


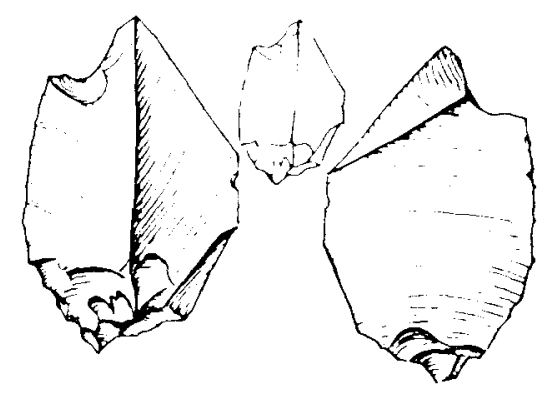

Fig. 9. Microbulino sauveterriano proveniente dallo strato A del la Sala Terminale (Scavi 1957-58). Disegno di B. Santochi. (Da P. Leonard, 1957, e A. Broglio in: P. LeOnARDi et alii, 1962).

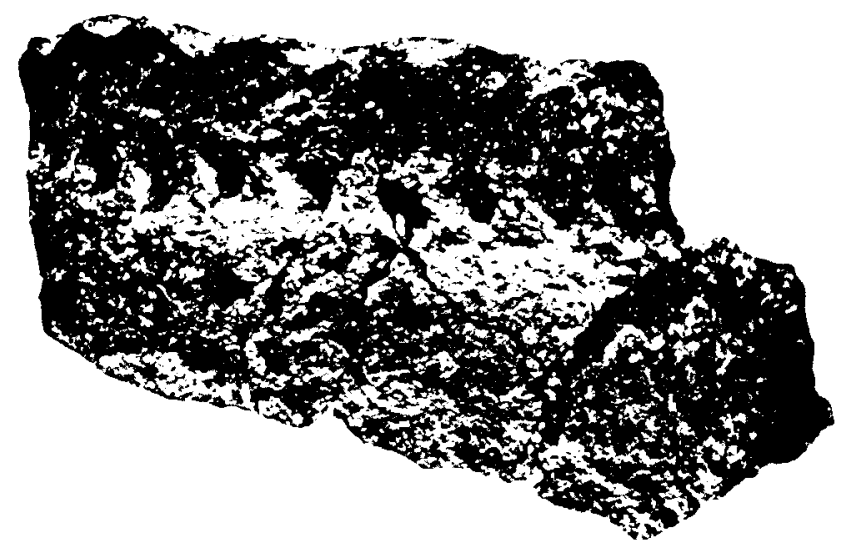

Fig. 10. Frammento di vaso fittile probabilmente calcolitico, proveniente dallo strato superficiale 4 della Grottina Azzurra. Due terzi della grandezza naturale. Fotografia di S. Borsetti. (Da A. Broglio in: P. LeONARDi et alii, 1962).

due coppie di incisioni trasversali parallele, evidentemente intenzionali, a metà circa del pezzo (fig. 12 b). Si può pensare, sia pure con riserva, che potesse trattarsi di un amuleto.

Più propriamente si può parlare di grafía a proposito di un reperto della campagna di Scavo dell'ottobre 1988, proveniente dallo strato $10 \mathrm{C}$ all'imbocco della Grottina Azzurra, nel quadrato 48. In questo strato sono stati reinvenuti alcuni manufatti probabilmente riferibili al Paleolítico Medio (Mustieriano?). Non è del tutto sicuro però questo riferimento per il nostro pezzo, essendo esso stato trovato nella parte contro-parete del deposito. 

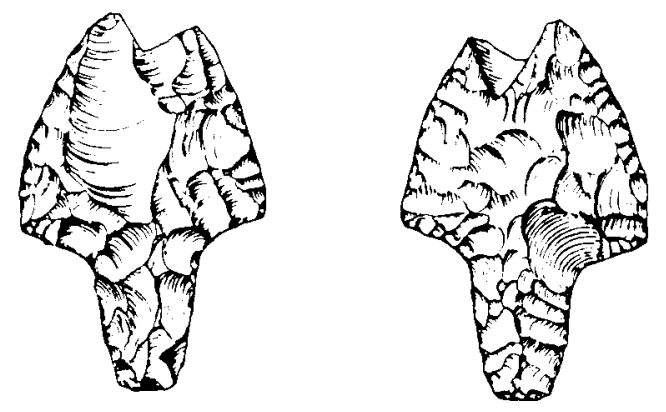

Fig. 11. Punta di freccia a ritocco piatto bifacciale, probabilmente calcolitica, proveniente dallo strato A della trincea antistante alla Grottiza Azzurra (campagne di scavo 1957-58). Grandezza naturale. Disegno di B. SANTOCHI. (Da A. Broglio in: P. LEONARDI et alii, 1962).

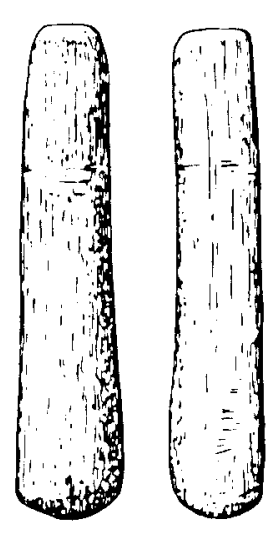

$(x$
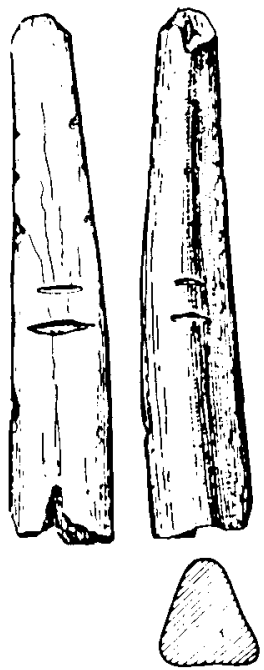
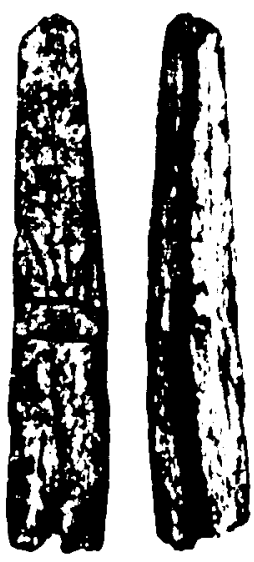

b

Fig. 12a. Manufatto osseo subcilindrico probabilmente riferibile al Gravettiano tardivo, rinvenuto al limite tra gli strati A' e A" nella Grotta di Trene, sempre sul versante sud-orientale dei Berici, non molto distante dalla Grotta di Paina. Grandezza naturale. Disegno di B. SANTOCHI (Da PIERo LeONARDI, 1959).

Fig. 12b. Frammento di os penis di Orso (speleo?) dello strato A della Grottina Azzurra (scavi 1957-58), con incisioni. Grandezza naturale. Disegno di B. SANTO$\mathrm{CHI} e$ fotografia di S. BoRSETti. (Da AAVV, 1988) 
Comunque si tratta di un osso metapodiale sul quale si scorgono chiaramente cinque brevi incisioni rettilinee trasversali subparallele, indubbiamente intenzionali (fig. 13).

Non è certo una gran cosa ne' dal punto di vista grafico, ne' tanto meno, da quello artistico. Tuttavia esso ráppresenta la più sicura testimonianza di un'attività per così dire "intellettuale" da parte dei cacciatori paleolitici che praticarono la Grotta di Paina.

\section{RIASSUNTO}

La Grotta di Paina è situata nei Colli Berici sul versante sudorientale, poco sopra il paese di Mossano. Scoperta nel 1939, e segnalata per la prima volta nel 1940, fu oggeto, a partire dal 1957, di regolari campagne di scavo, che sono tuttora in corso.

La Grotta consta di tre cavità collegate; la "Sala Grande" di notevoli dimensioni, la "Sala Terminale", che risponde alla sua parte più interna, e una piccola cavità laterale chiamata "Grotta Azzurra».

Il deposito, è in parte alluvionale, dovuto ad un ruscello avente origine da un "camino" verticale situato all'estremità posteriore della Sala Terminale, ora otturato, in parte constituito da materiali di crollo della grotta, in parte da apporti eolici esterni, loess. Esso non ha grande potenza, ma comprende un certo numero di strati, che hanno fornito abbondante materiale paleontologico, specialmente resti di orso speleo, che abitualmente doveva avere nella grotta la sua tana, e altri resti di macromammiferi, probabilmente prede di cacciatori, e micromammiferi, in prevalenza rispondenti a "boli» (rigetti di rapaci).

Del punto di vista culturale, la serie della grotta di Paina, pur nel suo limitato spessore è una delle più ricche del Veneto per quanto riguarda la successiones cronologica.

Essa infatti ha fornito - specialmente nella Grottina Azzurra- una serie di manufatti che da una industria basale, probabilmente riferibile al Paleolitico Medio (Mustieriano?) strati 12-10), attraverso un livello aurignaciano (strato 9), uno gravettiano (strato 7), uno con industria epigravettiana antica a crans (strato 6), uno con industria epigravettiana recente (strato 5), giunge a un deposito superficiale più o meno rimaneggiato (strato 4) contenente manufatti di varia età: ancora epigravettiano recente, Mesolitico e Calcolitico. 


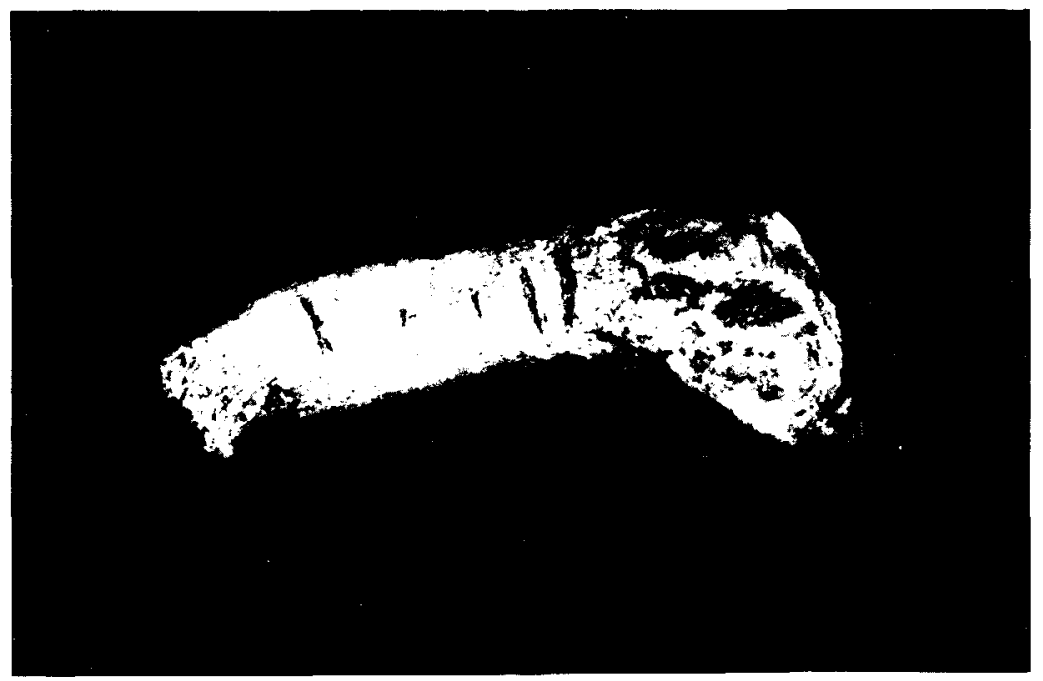

Fig. 13. Osso metapodiale di Orso speleo con incisioni trasversali parallele sicuramente intenzionali, rinvenuto nello strato $10 \mathrm{C}$ allimbocco della Grottina Azzurra, contenente manufatti probabilmente riferibili al Paleolitico medio. Grandezza naturale. Fotografía Mattiazzo.

Ripetiamo che la serie stratigrafico-culturale della Grotta di Paina è una delle più complete del Veneto - culturalmente parlando-per il $\mathrm{Pa}$ leolitico Superiore, e che addirittura, per quanto riguarda in particolare il'Epigravettiano antico italico a crans, la Grotta di Paina è la sola ad averlo rappresentato non soltanto nel Veneto, ma in tutta l'area padana.

Como si è detto sopra, lo studio palinologico, paleontologico a paletnologico dei reperti della Grotta di Paina concorda nel testimoniare la presenza dell'uomo nel Interpleniglaciale a nel secondo Pleniglaciale Würmiani, e in età olocenica.

Le caratteristiche a la distribuzione dei manufatti rinvenuti nella grotta suggerisce che essa nel corso del Paleolitico non sia stata usata come abitazione abituale, ma piuttosto come campo occasionale di caccia, e che lo sia divenuta soltanto in epoca recente, particolarmente nel Calcolitico. 


\section{BIBLIOGRAFIA}

AAVV, 1984: «l giacimenti dei Colli Berici». In: A. Broglıo et alii, «Paleolitico e mesolitico". Estratto dal Vol. / Veneto nell'antichità. Preistoria e protostoria, Verona.

AAVV, 1989: "Nuove ricerche nel deposito pleistocenico della Grotta di Paina sui Colli Berici (Vicenza)". Atti Ist. Ven. Sc. Lett. A. Tomo CXLVI (1987-88) - Cl. Sc. Fis. Mat. Natur. (1987-88).

Broglio, A., 1988: "Le grotte dei Colli Berici e la Preistoria", in: AAVV I Colli Berici natura e civiltà. Signum Edizioni.

Broglio, A. e LeONARDI, P., 1963a: "Les industries leptolithiques preaurignaciennes et gravettiennes en Italie». Bull. Soc. Mérid. Speleol. et Préhist., Voll. VI a IX, 1956-1959.

LeONARDI, P., 1951: "La stazione preistorica del Castellon del Brosimo sui colli Berici (Vicenza)». Ann. Univ. Ferrara, NS. Sez. IX, Vol. I. N. ${ }^{\circ} 2$.

- 1957a: «Prima segnalazione di manufatti microlitici di tipo mesolitico nella Grotta di Paina sui Colli Berici (Vicenza)». Atti Ist. Ven. Sc. Lett. A., Vol. 115.

- 1957b: «Premières découvertes d'industrie microlithique du type mésolithique dans la Grotta di Paina». Bull. Soc. Etud. Rech. Préhist. Les Eyies, N. ${ }^{\circ}$.

- 1959: "ll covolo fortificato di Trene nei Colli Berici orientali (Vicenza). Stazione preistorica con industria gravettiana". Bull. Paletnol. Ital., NS, Vol. 68.

- 1958: "Sulla grotte con industria gravettiana dei Colli Berici». Atti VIIP, Congr. Naz. Speleol. Como, 1956, Mem. IV. ${ }^{\circ}$, Tomo II.o.

- 1963b: «ll Paleolitico nel versante meridionale delle Alpi». Rend. Soc. Cult. Preist. Trent., Trento, $\mathrm{N}^{\circ} 1$.

LeONARDI, P. e Broglio, A., 1962b: “Le Paléolithique de la Vénétie». Ann. Univ. Ferrara, NS, Sez. XV. ${ }^{\circ}$, Suppl. Vol. I. 
- 1962-63: “ll Paleotitico superiore dei Colli Verici». Archeoloski Vestnik - Acta Archaeologica, Ljubliana, Vol. XIII,-XIV.

Leonard, P., Pasa, A., Broglio, A., Ronchetti, G., 1962a: «La stazione preistorica del covolo di Paina nei Colli Berici (Vicenza)". Riv. Sc. Preist. Vol. XV. ${ }^{\circ}$.

TREVISIOL, G., 1940: "Una nuova caverna preistorica scoperta nel vicentino". Vedetta fascista. 